\title{
Filming of zooplankton: a case study of rotifer males and Daphnia magna
}

\author{
Pierluigi COLANGELI, Adam CIEPLINSKI, Ulrike OBERTEGGER*
}

Research and Innovation Centre, Fondazione Edmund Mach (FEM), San Michele all'Adige, Italy

\begin{abstract}
Filming live organisms can give new insights into the hidden life of plankton. Accessibly priced digital cameras are now available for a large range of users. Here, we demonstrate the technical setup and workflow of using a single-lens reflex (DSLR) camera to film the behaviour of males of two rotifer species, Brachionus angularis Gosse (1851) and Keratella cochlearis Gosse (1851), and of the cladoceran Daphnia magna Straus (1820). Rotifers are cyclical parthenogens that produce males only under certain environmental conditions. Thus, knowledge on rotifer males is still limited because of their ephemeral nature and because they are often smaller than females. We filmed males of $\mathrm{B}$. angularis and $\mathrm{K}$. cochlearis with a DSLR camera connected to a compound microscope to better understand their morphology and behaviour in comparison to conspecific females. While written descriptions have their scientific value, seeing is complementary because everyone can verify what has been described. We made our videos publicly accessible through links connected to the paper. Our videos are, to our best knowledge, the first on males of $\mathrm{B}$. angularis and $\mathrm{K}$. cochlearis. Furthermore, we filmed the behavioural response of $\mathrm{D}$. magna to ultraviolet (UV) radiation with a macro lens attached to the DSLR camera. Approaches like this are valuable tools in environmental teaching. To see live organisms with one's own eyes may contribute to raising public awareness about the value of water resources and their hidden communities. In summary, filming can be a valuable tool to ignite scientific discussion, but the videos need an open-access platform where they can be referenced in a topic-related order.
\end{abstract}

Key words: Video-microscopy; males; Lake Tovel; Brachionus angularis; Keratella cochlearis; DSLR.

Received: July 2015. Accepted: November 2015.

\section{INTRODUCTION}

Since the pioneering work of Antonie van Leeuwenhoek (1632-1723) in developing microscopic biconvex lenses (Ford, 1982) microscopy has become a fundamental method for studying microorganisms. Microscopy has made significant advances, and by techniques such as scanning electron and transmission electron microscopy amazing details on morphological structures of organisms can be obtained. However, these techniques require fixed samples limiting the observation of live organisms. In fact, behaviour and morphology of microscopic organisms are currently described by text, schematic representations, and images.

Filming live organisms can give new insights into the hidden life of plankton. In the past decades, a few researchers (Gilbert, 1963; Coulon et al., 1983) recorded rotifers on video cassette support to study behaviour such as mating, speed, trajectory, and predation. However, this technique did not find a vast application probably because of the restricted possibility to edit the filmed material, difficulty in data sharing with a vast audience, and the physical space needed for storing the videotapes. Recently, digital single lens reflex (DSLR) digital single-lens translucent (DSLT), and electronic viewfinder interchangeable lens (EVIL) cameras have become popular among amateurs and professionals alike for photo shooting and filming; however, even though such digital cam- eras can be easily attached to a microscope (Fig. 1), researchers seldom use these cameras to film microscopic life and produce a video. A video gives the possibility to observe repeatedly the same scene by simply freezing or decelerating certain parts (i.e., frames). This allows observing in detail the behaviour and morphological structures of organisms that are generally examined in a fixed state. In fact, the traditional faunological approach of sampling planktonic organisms followed by fixation is still the most common one. Fixing plankton such as rotifers often leads to changing morphology because generally rotifers retract the corona and the foot when exposed to a preservative, and illoricate species especially often become sphere-like indistinct objects (Obertegger et al., 2006). This morphological distortion also holds true for male rotifers possessing a weak to no lorica (Ricci and Melone, 1998).

Rotifer males are rarely investigated because they only appear for a restricted period during the year and populations mainly reproduce parthenogenetically (Fontaneto and DeSmet, 2015). While males of Bdelloidea have never been observed, rotifers of the subclass Monogononta show sexual dimorphism (Wallace et al., 2006). Rotifer males have: i) a rudimentary digestive apparatus (Pontin, 1978) because they do not feed; ii) are considerably smaller than females (Ricci and Melone, 1998); iii) have a shorter live-span than females (Gilbert, 1963); and 
iv) show a weak to marked resemblance to females (Fontaneto and DeSmet, 2015).

Here, we show how to easily use a DSLR camera to observe live zooplankton. We underline the utility of sharing videos within the scientific community to display and discuss the behaviour and morphology of microscopic organisms captured in videos. Open-access platforms, such as the well-known sites YouTube or DailyMotion may be suitable to share observations obtained in the laboratory with the scientific and lay community. We focused on rotifer males of Brachionus angularis and Keratella cochlearis to demonstrate the technical setup and workflow. Apart from descriptions and drawings of morphology and behaviour of rotifer males (Gilbert, 1963; Sudzuki, 1964; Pontin, 1978; Dumont et al., 2006; Fontaneto and DeSmet, 2015), there are, to the best of our knowledge, very few videos on rotifer males (see https://www.youtube.com/watch?v=F61cHnGih54). Our videos, which are accessible on YouTube to a vast audience, apart being the first for males of these species, also show details on morphology, swimming, and interaction of males with females. Furthermore, we filmed the behaviour of young individuals of Daphnia magna in response to ultraviolet radiation (UVA; 320-400 nm). Cladocera are known to avoid UVA radiation by actively swimming in the opposite direction (Hylander et al., 2014). The small experimental setup we describe can be readily used in environmental teaching and used for several purposes such as explaining and promoting scientific research.

\section{METHODS}

Brachionus angularis (Gosse, 1851) and $K$. cochlearis (Gosse, 1851) were isolated from Lake Tovel (Italy; $\left.46^{\circ} 15^{\prime} \mathrm{N} 10^{\circ} 56^{\prime} \mathrm{E}\right)$ and cultivated in petri dishes with WMC culture medium (Guillard and Lorenzen, 1972) under a $14: 10$ light/dark cycle at $13.5^{\circ} \mathrm{C}$. Cultures were fed weekly with Chlorella vulgaris (Scandinavian Culture Collection of Algae and Protozoa strain K-1801).

Males of $B$. angularis and $K$. cochlearis, respectively, were placed together with conspecific females using a

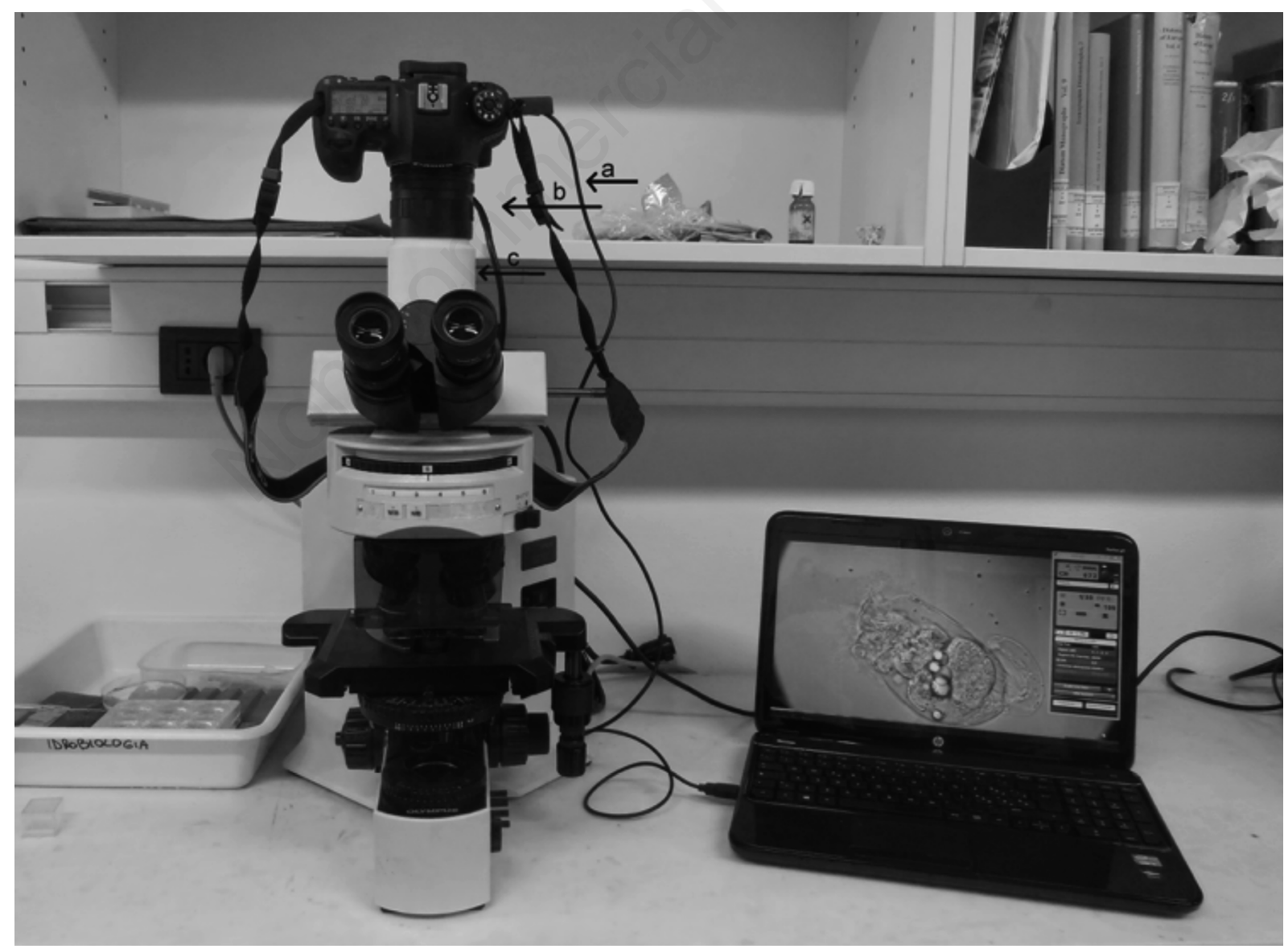

Fig. 1. Microscope with attached DSLR camera connected to the computer via USB cable (a) for live-preview filming; please note that the camera's objective lense was removed and that the camera was connected by an extension tube (b) to the microscope. A plastic adaptor (c) held the extension tube in place. 
glass pipette on an ad hoc swimming chamber (Fig. 2; description is given below), and their behaviour was filmed with a DSLR camera (Canon 6D full-frame digital camera shooting at 25 frames per second) connected to an Olympus BX-51 compound microscope. We would like to underline that any less expensive DSLR camera can also be used. Instead of the official Olympus camera adapters, an extension tube set was used to couple the camera to the microscope (Fig. 1). Here we assembled a $51 \mathrm{~mm}$ unbranded generic extension tube by screwing together three threaded pieces $(7,14,28 \mathrm{~mm})$, and fixed the tube to the microscope by a plastic cylinder (Fig. 1). Longer tube length gives higher resolution but reduces the field diameter. To ease the workflow of filming, we used the Canon Utility software to connect the camera to a computer to remotely control the settings and to view in live-preview mode on the computer monitor (Fig. 1). The first $30 \mathrm{sec}-$ onds of the video on B. angularis (video 1) were filmed under a stereomicroscope (Wild Macroscope M420) and the remaining time under a compound microscope (Olympus BX-51) with differential interference contrast (DIC) light settings to gain adequate contrast. In video 2 (video 2), filmed using the compound microscope, the first 1:06 minutes show a live female of $B$. angularis in the swimming chamber while afterwards the female is on a glass slide. Video 3 (video 3) shows K. cochlearis with the same setup as video 1 . When filming the swimming chamber, we used the 20x/0.5 UPlanFI Olympus objective lens, and, when we used glass slides in combination with a cover slip we used the $40 \mathrm{x} / 0.75$ UPlanFI and the 60x/0.90 UPlanAp Olympus objective lenses (as indicated in the video).

The swimming chamber (Fig. 2) was built according to Coulon's et al. (1983) general design. We used a glass microscope slide as the base of the swimming chamber, upon which four glass coverslips were glued with cyanoacrylate glue to form a chamber $(2 \times 2 \times 0.25 \mathrm{~mm})$ with a total volume of approximately $1 \mathrm{~mm}^{3}$ (Fig. 2). The swimming chamber gave the necessary three-dimensional space for normal swimming behaviour such as making flips, revolutions and loops, while at the same time kept the specimen within the field of vision. In filling the chamber with specimens and culture medium, we tried to prevent the formation of a water meniscus, which induces chromatic aberrations and perspective distortions, by adding as little water as possible; no cover slip was used. Unfortunately, rotifer males are quite delicate and a compromise had to be found between the amount of water added and survival of males. Because our focus was on filming male - female interactions, an inferior image quality was acceptable considering we were interested in gross scale morphological details. In fact, the height of the chamber was too thick for perfect image quality with respect to the size of the rotifer males and females of $K$. cochlearis and B. angularis (maximum length $200 \mu \mathrm{m}$ ), and thus morphological details were obtained by placing specimens on a glass slide.

Daphnia magna neonates ( $\leq 24$ hours old) were obtained from a laboratory culture and exposed to UVA $\left(14.6 \pm 4.5 \mathrm{~W} \mathrm{~m}^{-2}\right)$ radiation produced by UVA LEDs $(3 \mathrm{~V}$, $20 \mathrm{~mA}$ ). UVA radiation was measured with a DeltaOhmHD2102.1 radiometer. Three UV radiation transparent cuvettes were used as experimental chambers (Fig. 3). Each cuvette, with $5 \mathrm{~mL}$ of culture medium, received five neonate Daphnia specimens. The neonates were exposed to five cycles of 15 seconds (") of UVA radiation inter-

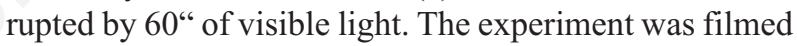
(video 4) with a $180 \mathrm{~mm}$ macro lens.

\section{Video editing}

Several video editors exist; some are released with proprietary licence (e.g., Adobe Premiere, Sony Vegas), whereas others are released as freeware (e.g., Lightworks). To produce the final video, we edited the raw material using basic operations such as: i) pan/crop to move and zoom to the point of interest; ii) text overlay to indicate specific morphological characters; iii) time stretches

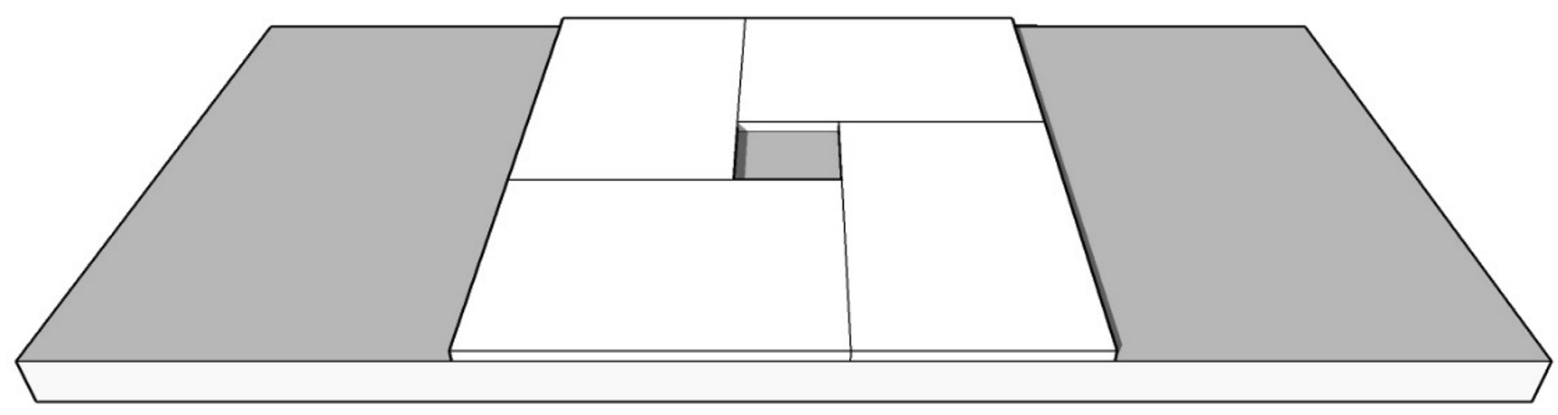

Fig. 2. Swimming chamber; note the alternate displacement of the coverslips. 
to slow down or accelerate un-interesting frames/scenes; iv) fading controls to obtain a smoother transition between different scenes; v) brightness/contrast controls to maximise the sharpness of the images.

\section{RESULTS AND DISCUSSION}

The videos confirmed most of the characters described in dedicated keys or zoology texts (Sudzuki, 1964; Pontin, 1978; Koste, 1978; Fontaneto and DeSmet, 2015) on rotifer males.

\section{Brachionus angularis (video 1)}

The initial sequence (time 4-30") shows the swimming activity of $B$. angularis. Males swim considerably faster (27") and are smaller (50") than females. An overview of the three-dimensional structure of the male can be seen by observing the rotating male (55"). We tentatively suggest that we can see moving spermatozoa (1'14") in the posterior part of the body. According to Sudzuki (1964), the foot in B. angularis is retractable while Pontin (1978) is not clear about this. Our film clearly shows the ability of a $B$. angularis male to move its foot in and out from the delicate lorica (1'30"), par- tially exposing the non-turgid penis (2'05"). Our videos also offer the opportunity to observe (1'43") a brownish spot located over the prostatic gland in posterior dorsal position in a living male that we link to excretion granules as described by Fontaneto and De Smet (2015). Furthermore, sensory bristles (1'52") can be seen, which are probably used in female recognition and sensing of the environment (Schmidt-Rhaesa and Kükenthal, 2015).

Males and females were put together several times but we were not able to observe any mating behaviour, except for one occasion (2'15'). This difficulty in observing mating behaviour is in contrast to Gilbert (1963) and Gómez and Serra (1995) who describe an immediate mating between males and mictic or amictic females. However, Gilbert (1963) states that young males are more successful than old ones. Probably, we used older males for our filming of male-female interactions or we missed mating because of the time necessary to adjust the film settings. For the only interaction between a male and a female (2'15"), we hypothesise that we observed the last sequence of the mating behaviour as described in Gilbert (1963): the male drags behind the female. Our video shows (2'30") that the sagittal plane of the female was not in the same direction of the movement, indicating that the male was responsible

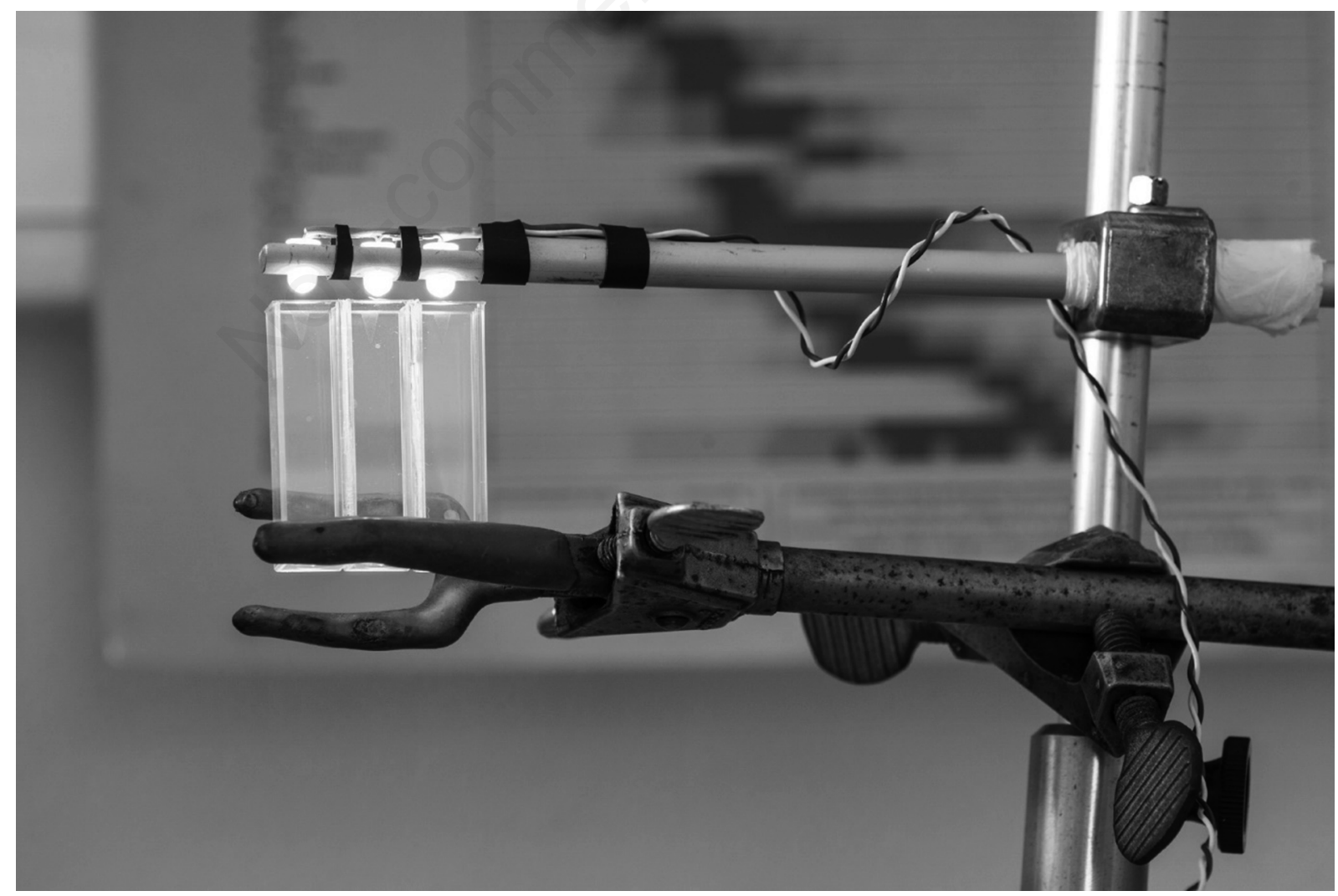

Fig. 3. Daphnia magna film setup: the three cuvettes are exposed to UVA radiation from above. 
for the swimming direction by dragging behind the female. Furthermore, the video shows a thread-like structure linking male and female. Gómez and Serra (1995) report on a fine thread between the mating pair, but the nature of this structure is unclear. In any case, further videos are needed to give more details on the complex mating behaviour of $K$. cochlearis.

We also filmed a live female of $B$. angularis (video 2) in the swimming chamber (1'06') and afterwards on a glass slide. In filming the female, we changed the DIC settings to find the best contrast for certain structures such as trophi. We kept this sequence in the video to show how the quality of the video changes accordingly to the shift of the Normaski prism. In the video, we highlighted several morphological features otherwise difficult or impossible to observe in a fixed state. The dynamic movements of the corona, trophi, intestine, and foot might not only fascinate rotiferologists but also the public. In our video, not only the foot and the malleate trophi but also the muscles that control the foot can be seen in action. Recently, the fine-scale movement of the trophi has attracted attention (Hochberg et al., 2015), and videos such as ours can be the first step to investigate in vivo trophi mechanics. Furthermore, the three-dimensional structure of the corona and the body of $B$. angularis can be more easily grasped by our video than by photos or drawings. Towards the end of the video, we can see the dying rotifer female that starts to lose the cilia of the corona.

\section{Keratella cochlearis (video 3 )}

The initial sequence (1-26") gives an overview on sexual dimorphism in $K$. cochlearis and shows that, similarly to $B$. angularis, males are smaller (approximately $50 \mu \mathrm{m}$ ) and swim faster than females. As shown in Voigt and Koste (1978), males lack spines and have a permanently exposed foot (27-50"). Interestingly, the body of a $K$. cochlearis male lacks the brownish granules seen in $B$. angularis. The rotation of the male (44-55") gave an impression of the three-dimensional structure. Our video clearly shows that also males of $K$. cochlearis are able to retract their foot (1'12") and possess cilia on the foot (1'40"). Noteworthy is the constriction under the head (1'25') that might be a sign of stress under laboratory conditions.

\section{Daphnia magna response to UVA (video 4)}

The video starts by showing three cuvettes with $D$. magna neonates exposed to normal light. Individuals swam stochastically and occupied the entire space. Subsequently, by exposing the upper part of the cuvette to UVA radiation ( 1 '00"), individuals swam to the bottom of the cuvette seeking refuge from UVA radiation. After switching off the UVA radiation (1'15"), the neonates again showed normal swimming behaviour, reaching the middle-high portion of the cuvette after a few seconds. The cycle of switching on and off UVA radiation was then repeated four times. This simple setup showed in a straightforward way how zooplankton escapes harmful UVA radiation. In order to do so, zooplankton must sense the threat (e.g., see Smith and Macagno, 1990), and this topic can be easily discussed in the classroom or with the public by using a simple setup as the one shown.

\section{CONCLUSIONS}

The observation of microorganisms is still as fascinating now as it was at the time of Antonie van Leeuwenhoek, when he was visited both by curious and sceptics (Ford 1982). Filming microinvertebrates with a DSLR camera gave us the opportunity to see what others described in writing. Our methodological setup does not require specific technical skills and can be easily extended to filming other microorganisms such as algae or protists. In any case, the filming of moving organisms poses some challenges and requires some testing to find the right settings. While schematic drawings and text have their place in a scientific context, seeing is complementary to describing. Nowadays, we can easily disseminate videos on open-access platforms such as YouTube, DailyMotion, vimeo.com or ZippCast that try to serve the preferences of their users, and thus can reach a vast audience. From a scientific point of view, it would be advisable to find a dedicated database such as http://rotifera.hausdernatur.at/ that could review and host subject-specific links to videos to guarantee their scientific standard and facilitate their dissemination. A dedicated platform could also encourage discussion between researchers on their observations. From a citizen science perspective, filming allows showing an interested public the fascinating life of microorganisms that is generally accessible only to researchers. The ecological importance of plankton is especially neglected by the public, and videos such as these could attract attention and raise public awareness.

\section{ACKNOWLEDGMENTS}

The first author was funded by the European Social Fund (P.O. FSE Abruzzo 2007-2013 - SG/RI/WE/98) and thanks the Edmund Mach Foundation for hosting him. We thank Giovanna Flaim for improving the manuscript. We thank Scott Mills, an anonymous reviewer, and the handling editor Diego Fontaneto for their suggestions.

\section{REFERENCES}

Coulon P, Charras J, Chassé J, Clément P, Cornillac A, Luciani A, Wurdak E, 1983. An experimental system for the automatic tracking and analysis of rotifer swimming behaviour. Hydrobiologia 104:197-202.

Fontaneto D, DeSmet WH, 2015. Rotifera, p. 217-301 In: A. 
Schmidt-Rhaesa and W. Kükenthal (eds.), Gastrotricha and Gnathifera. De Gruyter, Berlin.

Ford BJ, 1982. The Rotifera of Antony van Leeuwenhoek. Microscopy 34:362-373.

Gilbert JJ, 1963. Contact chemoreception, mating behaviour, and sexual isolation in the rotifer genus Brachionus. J. Exp. Biol. 40:625-641.

Gómez Á, Serra M, 1995. Behavioral reproductive isolation among sympatric strains of Brachionus plicatilis Müller 1786: insights into the status of this taxonomic species. Hydrobiologia 313:111-119.

Guillard RR, Lorenzen CJ, 1972. Yellow-green algae with chlorophyllide C. J. Phycol. 8:10-14.

Hochberg R, Wallace RL, Walsh EJ, 2015. Soft bodies, hard jaws: an introduction to the symposium, with rotifers as models of jaw diversity. Integr. Comp. Biol. 55:179-192.

Hylander S, Ekvall MT, Bianco G, Yang X, Hansson L-A, 2014. Induced tolerance expressed as relaxed behavioural threat response in millimetre-sized aquatic organisms. P.R. Soc. B 281:20140364.

Koste W, 1978. [Rotatoria: die Rädertiere Mitteleuropas].[Book in German]. Gebrüder Borntraeger, Berlin: pp. 673.

Obertegger U, Braioni MG, Arrighetti G, Flaim G, 2006. Trophi morphology and its usefulness for identification of formalinpreserved species of Synchaeta Ehrenberg, 1832 (Rotifera: Monogononta: Synchaetidae). Zool. Anz. 245:109-120.

Pontin R, 1978. A key to British freshwater planktonic rotifera. Freshwater Biological Association, Ambleside: 178 pp.

Ricci C, Melone G, 1998. Dwarf males in monogonont rotifers. Aquat. Ecol. 32:361-365.
Smith KC, Macagno ER, 1990. UV photoreceptors in the compound eye of Daphnia magna (Crustacea, Branchiopoda). A fourth spectral class in single ommatidia. J. Comp. Physiol. A 166:597-606.

Sudzuki M, 1964. New systematical approach to the Japanese planktonic Rotatoria. Hydrobiologia 23:1-124.

Wallace RB, Snell TW, Ricci C, Nogrady T, 2006. Rotifera - Biology, ecology and systematics. In: H. Segers (ed.) Guides to the identification of the microinvertebrates of the continental waters of the world. Backhuys Publ., Leiden: 295 pp.

\section{VIDEO LINKS}

Colangeli P. [pierluigi colangeli]. (2015, July 27). video 1 - Brachionus angularis Gosse (1851) [Video file]. Retrieved from http://youtube.com/watch?v=77IRcrQenRQ

Colangeli P. [pierluigi colangeli]. (2015, July 29). video 2 - Brachionus angularis Gosse (1851) female [Video file]. Retrieved from http://youtube.com/watch? $\mathrm{v}=\mathrm{SS}-\mathrm{UWbCEb} 4 \mathrm{~s}$

Colangeli P. [pierluigi colangeli]. (2015, July 27). video 3 - Keratella cochlearis Gosse (1851) [Video file]. Retrieved from http://youtube.com/watch?v=IgkMGkA0kUU

Colangeli P. [pierluigi colangeli]. (2015, July 28). video 4 Daphnia magna UVA response test [Video file]. Retrieved from http://youtube.com/watch?v=kcqh2KU4fYk

Hotos G. [George Hotos]. (2015, May 23). Dr G. Hotos Lab-GreeceRotifers, Brachionus plicatilis, male [Video file]. Retrieved from http://youtube.com/watch?v=F61cHnGih54 